from the fact that the inflammation is seated beneath the deep cervical fascia. This fascia consists of several layers, the undermost of which is continuous with the pericardium. Anatomists tell us that the fascia varies in different bodies. Is it fair to surmise that the danger in some cases is very great owing to the thickness and firm attachments which this fascia then presents?

old Cavendish-street, $W$.

\section{CASE OF DISEASE OF THE AORTIC VALVES PRESENTING SOMEWHAT UNUSUAL PHYSICAL SIGNS.}

\author{
BY DAVID DRUMMOND, M. A., M.D., \\ PHYSICIAN TO THE NEWCASTLE-ON-TYNE INFIRMARY.
}

R. D-, aged twenty-nine, a carman, was admitted into the Newcastle-on-Tyne Infirmary on the 26th of June last, giving the following account of his illness. He had been in excellent health previous to the close of last year, but, owing to exposure during the very severe weather, whilst following his employment, towards the end of December he was laid up with a cold, which resulted in attacks of pain in the chest. (What the exact character of this initial pain may have been it is impossible to arrive at.) A few weeks subsequently the pain assumed a spasmodic nature, coming on during exertion, and lasting from fifteen to twenty minutes. About this period-i.e., a month after the commencement of the attack-he began to be troubled with beating in the chest, which has gradually increased up to present. One month previous to admission he became aware of a "rubbing" accompanying the beating, and at the same time could hear a "loud rasping noise going on in his chest." He has never been at all troubled with shortness of breath, not even when running up-stairs; coughs infrequently, without expectoration; has never had hæmoptysis nor suffered from rheumatism. He is a tall and somewhat spare man, with an anxious expression of face, evidently caused by the repeated attacks of angina pectoris, which had prompted him to seek relief in the hospital.

On stripping his chest, my attention was at once called to the violence of the pulsation, cardiac and arterial. Almost the entire thorax anteriorly, especially the left side, heaved in a tumultuous manner; the arteries of the neck and the subclavians coursing beneath the clavicles also pulsated with great force. An extremely rough and rasping thrill "frémissement cataire" was communicated to the hand when placed on any part of the front of the chest, but was best marked in the left parasternal line, corresponding to the second and third interspaces. This thrill was distinctly diastolic in rhythm, and was accompanied by a loud harsh murmur, best heard in the pulmonary area and to the middle of the sternum, so loud as to be easily audible six or eight inches from the chest-wall without the stethoscope, and distinctly heard by the patient himself. This diastolic murmur, though audible all over the thorax, became com paratively feeble in the mitral area and round to the axillary line, but particularly loud between the shoulders, and especially to the right side of the spine, and could be heard as a loud harsh murmur in the carotids, subclavians, abdominal aorta, and left femoral. A murmur with the first sound was also present, loudest at the top of the sternum, but very faint in the mitral area, and distinctly audible in the vessels before mentioned, making a double arterial murmur. The heart was much hypertrophied, the cardiac area of dulness being markedly increased. On the left parasterna line, dulness commenced at the lower border of the second rib, and was lost downwards in the liver dulness; in a horizontal direction, on a level with the fourth rib, it extended from the right parasternal line to the axillary line. The pulse at the wrist was visible and distinctly "water-hammer" in character, but the superficial vessels themselves were free from atheromatous disease. He complained very much of the attacks of angina, three or four of which he experienced each day. Physical examination of the lungs revealed nothing abnormal. The urine was free from albumen, and a fair quantity was passed each day. There was no dema of the ancles or elsewhere ; in fact, as he himself remarked, "But for the constant beating in my chest, and the noise up into my ears, which is like to open my head, and the pain, I have never been in better health in my life." Now and again there was a "tumble" in the beat of the heart, as if an aborted systole had occurred; this intermission was very infrequent, about once in every two minutes. The enormous percussion stroke and huge tidal wave in the sphygmographic tracing, indicating very great hypertrophy of the left ventricle, with a free (for exit) aortic orifice, the dicrotic wave being nearly abolished, it is probable there is a good deal of regurgitation.

The patient was treated by considerable doses of liquor arsenicalis (Fowler's solution) seven drops for the dose three times a day, with fifteen drops of tincture of digitalis. The attacks of angina were soon arrested and kept in abeyance by the arsenic, and the patient left the infirmary very much relieved, though still complaining of the violence of the pulsations, and the loudness of the noise in the head.

Remarlis.-The case is of interest,-lst, as being an example of aortic regurgitation presenting the signs of extremely harsh and loud murmur with rasping thrill. 2nd. There was undoubtedly very considerable regurgitation back into the left ventricle, without any constitutional disturbance ; the evidence of the large amount of regurgitation being $(a)$ double murmur in the arteries (Balfour's "Diseases of the Heart "); (b) great hypertrophy ; and (c) obliteration of the dicrotic wave in pulse-tracing. 3rd. The case shows well the great value of arsenic in angina pectoris ; in all the cases I have used it, pushing the drug up to ten or twelve drops of Fowler's solution for the dose, there has been marked benefit.

Newcastle-on-Tyne.

\section{THE CONDITION OF THE CERVIX UTERI IN CASES OF PLACENTA PRAVIA.}

BY GEORGE ROPER, M.D.,

PHYSICIAN TO THE EASTERN DIVISION OF THE ROYAL MATERNITY CHARITY, LONDON.

IN the mind of everyone engaged in the practice of difficult midwifery, placenta prævia has always occupied a place of importance. Its treatment, in my own estimation, is not quite clear, but I am not going to enter on the management of this anxious form of complex labour at present. I will confine my remarks to the observation which I wish to record. I will not say that it has hitherto been unobserved, but, so far as I can learn, no description of it has as yet been published. I have the permission of my friend Dr. Barnes, whose knowledge of the literature of the subject is second to none, to say that he is unacquainted with any such record in English or foreign medical writings.

The condition to which I refer, as a clinical fact, consists in a peculiar induration of the os or cervix uteri, at the site of placental attachment, dependent on an alteration of the uterine texture of that part on which the placenta is implanted. From clinical observation it may be said, that wherever the placenta grows, whether on the fundus or elsewhere on the inner uterine surface, the area of attachment is marked by induration and thickening of the uterine tissue. It is a common belief that the structure of the cervix uteri in placenta prævia is soft and easily dilatable, and is rendered more so by the profuse bleeding which usually happens in these cases. Leishman, in the second edition of his "System of Midwifery," p. 439, says: "We shall probably find that the os and cervix uteri are somewhat peculiar to the touch. This peculiarity consists in a doughy feeling, due to the unusual thickness of the cervix, which is necessarily permeated with large vessels for the placental circulation." And again, at p. 446, he says, in speaking of the operation of podalic version in placenta prævia: "During the course of this procedure, which is often easier of execution than under ordinary circumstances, owing to the relaxed state of the uterus, the result of hæmor. rhage." Schroeder, in his " Manual of Midwifery," translated by Dr. Carter (Sydenham Society), p. 312, remarks on the introduction of the hand into the uterus for the purpose of effecting podalic version in placenta provia: "The lower 
uterine segment being generally soft and yielding in placenta provia, this often succeeds very early." In my own experience the clinical fact has been that induration and thickening corresponding to the extent of placental attachment have been unusually great, and the induration has eonstituted one of the greatest difficulties in the way of safely effecting forced delivery.

If we have to deal with a case of placenta prævia in which the placenta is centrally placed, then there is induration in the entire circumference of the inner os; if with one of a partial kind, then we have a demonstrative proof, in that we are able to compare that part of the os internum to which the placenta is attached, with that portion to which it is not attached; the former will be found indurated and unyielding, the latter soft and elastic. The following report will give an illustration of this condition.

0n the 22nd of May, 1879, a midwife of the Royal Maternity Charity requested my attendance on a patient, aged twenty-nine, in labour with her fifth child, at the end of the eighth month of pregnancy. She had had much flooding, but not to such an extent as to constitute present danger. The os uteri admitted two fingers. The placenta was partially over the os, and was attached to the anterior segment of the uterus. At the posterior edge of the placenta I could feel the foetal head, and between the head and the symphysis pubis was one of the feet; a loop of pulseless funis was hanging down through the os. Had there been any imminent dange to the mother, necessitating immediate delivery, the obvious course would have been to perforate the head. With two fingers passed through the os uteri for the purpose of artificially detaching the placenta, the foot was seized and brought down, and the child easily turned. The os uteri was not sufficiently dilated nor dilatable enough to allow of extraction. I therefore pulled the breech of the child down only so far and so forcibly as to test its dilatability. The condition of the os uteri at once afforded evidence of induration and roughness of surface and inelasticity of its anterior portion to which the placenta had been attached, while the posterior segment which had not been implicated in the placental attachment was smooth, soft, thin, and elastic. As there were no labour pains, I determined not to use any further effort, for the present, to deliver; but directed the midwife to use gentle traction in co-operation with labour pains when they occurred. I left the patient at $6 \mathrm{~A} . \mathrm{M}$., and visited her again at $10 \mathrm{~A}$.M. There had been no labour pains. I passed my finger up between the breech of the child and the margin of the os uteri, to ascertain whether the os had become more dilatable; I thought it had, and applied a sufficient tractile force to bring the foetal chest into the os uteri, when the tension of the rigid part of the os became too great to justify proceeding further. As she had scarcely any labour pains, I injected, subcutaneously, one grain of ergotine, and again left her in charge of the midwife. At 2 P.M., in company with Dr. Herman, I saw the patient again. The child had now been turned eight hours and although its position seemed not to produce any injurions pressure, and the mother was not in any respect suffering much, yet I thought it desirable to deliver her. Dr. Herman concurred with me in what I have described as to the condition of the os and cervix. In spite of the great care and gentleness with which traction was used in liberating the head, the indurated portion of the cervix sustained a transverse laceration. At this stage, some one may say that the base of the cranium should have been perforated and delivery effected by the cephalotribe; but this is a point $I$ am not now discussing.

I do not wish to be understood as overlooking the undeveloped state of the lower part of the uterus in these cases, occurring as they mostly do before full term; and in speaking of the os or cervix uteri, I mean the os or margin of the orifice of the uterus proper. A great distinetion is to be drawn between the os internum, which is firm and thick, and the thin flabby cervix with its soft outer os.

While engaged in writing this paper, I was called to see a patient in the charity who had had flooding for a fortnight. she was thirty-six years old, and at the end of the eighth month of pregnancy with her fifth child. Although she had lost much blood, she had a good pulse, and was not much blanched. She had no symptoms of immediate danger. The os uteri was open so as to admit two fingers. The placenta was nearly centrally placed, but more posteriorly than anteriorly, for I could pass the finger in front of the anterior margin of the placenta and feel the foetal head; posteriorly I could not reach the border of the placenta.
The placenta had to a large extent become spontaneously separated, and no hæmorrhage was going on at this time, now twelve at noon. There was marked hardness, thickness, and roughness of surface all round the os internum, but more intense on its posterior segment, to which the placenta had been more extensively attached. As theflooding had ceased, I left her in charge of the midwife, hoping that, as pains came on, natural delivery would follow. I promised to see the patient again during the afternoon. At 4 P.M. the midwife became alarmed by the return of slight hæmorrhage, and sent for Mr. Lane, one of my colleagues in the charity; he kindly attended immediately, and delivered by podalic version. I had made an appointment for Dr. Herman to see her with me, for the purpose of observing the condition of the os uteri, but on our arrival she had been delivered about a quarter of an hour. I was somewhat disappointed that we had not again an opportunity of making this observation before delivery. An examination, however, soon after delivery, afforded marked evidence of the induration I have referred to ; it was greater posteriorly, corresponding to the site at which the placenta had been more extensively situated; it entirely belonged to the os internum, or substance of the uterus proper, for the whole of the cervix, with its os externum, was soft and pulpy. When Mr. Lane's attention was called to it, he remarked, "I had observed it and thought there was a diseased condition of the cervix, which might give future trouble." I was therefore glad that it had made such a forcible impression on him, quite independently of any suggestion from either of us.

$I$ have already said that wherever the placenta is im. planted the uterine texture at the spot undergoes thickening and induration, and the surface of the placental area is rough. These conditions are to be observed whenever the hand has to be introduced into the nterus after delivery ; it matters not on what part of the inner uterine surface the placenta area may be. We see them also in post-mortem examinations. In this subject there is a fine field for histological research. An interesting paper by Dr. John Williams has been published in the Obstetrical Society's Transactions, vol. $\mathrm{xx}$," "On Some of the Changes in the Uterus resulting from Gestation," \&c., from which I will make a short quotation, as bearing on this topic:- "The veins or sinuses are enlarged throughout the body of the uterus during gestation, but the enlargement is far greater in that part of the wall to which the placenta is attached. This, when cut into towards the end of pregnancy, presents almost a trabeculated structure, so completely riddled is it with anastomosing venous channels of considerable size." Much might be said on the importance of this condition in reference both to the treatment of placenta prævia and its prognosis after forced delivery; but this would be going beyond the object of this paper.

West-street, Finsbury-circus.

\section{STONE IN THE BLADDER OF A PATIENT} EIGHTY-EIGHT YEARS OLD;

BIGELOW'S OPERATION; SUCCESS PERFECT.

BY W. F. TEEVAN, B.A., F.R.C.S., SURGEON TO THE WEST LONDON AND ST. PETER'S HOSPITALS.

ON September 9th, in consultation with Dr. Kempe, of Ladbroke-grove-road, I saw Colonel —, eighty-eight years of age, who served throughout the Peninsular war. From notes taken by Dr. Kempe, it appeared that the patient passed two calculi many years ago. For a long time he had occasionally suffered from pain in the loins, and had seen red gravel in his urine. One year ago the gravel ceased to appear, and he began to be troubled with a frequent desire to micturate, and to have a dull aching pain iu the perineum. Now and then he saw a little blood in his urine. Latterly his pains had increased, and the water had become very thick and offensive. For the past three months he had been confined to bed. During the last few weeks he hall become very excitable and unmanageable. He could get but little sleep at night, as he was so frequently troubled to urinate. 